Section 2150

\title{
Undergraduate Research Projects for Engineering Technology Students
}

\author{
Chih-Ping Yeh, Jeannie Drew, Chris Rockwell, Hai-Chun Chien \\ Division of Engineering Technology \\ Wayne State University
}

\begin{abstract}
This paper describes three undergraduate research projects conducted in the Division of Engineering Technology at Wayne State University. All of the three projects were supported by the WSU Undergraduate Research Grants. The research problems, approaches taken, and respective roles of the students and faculty member are presented. The impact of undergraduate research on ET education and future challenges are discussed.
\end{abstract}

\section{Introduction}

Research experience for undergraduates is of great importance not only for conducting research on a topic that has impact on a current research activity, but also as a tool to enhance undergraduate education $^{1-3}$. For the engineering technology students, research experiences allow them to carry out in-depth study of engineering concepts, while emphasizing hands-on experiences and practical applications. Participating in funded research projects strengthens the student's resume, and fulfills the requirements of present day employers, who demand sound engineering skills in their employees.

At Wayne State University (WSU), the Undergraduate Research Council funds up to twelve proposals each semester. All undergraduate students, except graduating seniors, are invited to submit research proposals for competition. The proposed research project must be sponsored by the faculty and involve the undergraduate working in collaboration with a faculty member. Each proposal, limited to four pages, must include a project description, research problem(s), roles of the faculty and student, proposed project duration, budget justification, and a letter of recommendation from the faculty. A budget of $\$ 2,000$ is available for the student, either as a tuition or stipend, and $\$ 500$ is available for justified research cost. At the end of the project period, the student or the faculty must submit a final report on the research to the Undergraduate Research Council. The report, limited to three pages, must outline the project's findings and the respective roles of the student and the faculty. It is suggested by the Undergraduate Research Council that each project be completed in no more than two semesters. For continued research, the original proposal can be resubmitted in the following semester for competition. This allows the student to earn up to $\$ 6,000$ in an academic year.

The Division of Engineering Technology (DET) at WSU has actively participated in 
the undergraduate research program since it was announced. On average, two to three proposals submitted by ET students are funded each semester. This paper presents three funded projects conducted during 2002. We selected these three projects to demonstrate the typical types of research projects carried out by the undergraduate students majoring in Electrical Engineering Technology at WSU-DET.

\section{Project Descriptions}

In this section we present describe research problems and the approaches taken in these three projects. Student participation in all three projects included literature research, circuit design, computer programming, laboratory set-up, and data measurement and analysis.

\section{a. Development of Calibration Test Bench for Electronic Components}

This is an industrial based project co-sponsored by the local automotive industry. The objective of this project is to build a calibration test stand whose foremost feature is the calibration check of electronic components that interface with data acquisition devices. The purpose is to test expensive automobile components, such as pressure transducers, for re-use. Figure 1 shows a test stand used in this project.

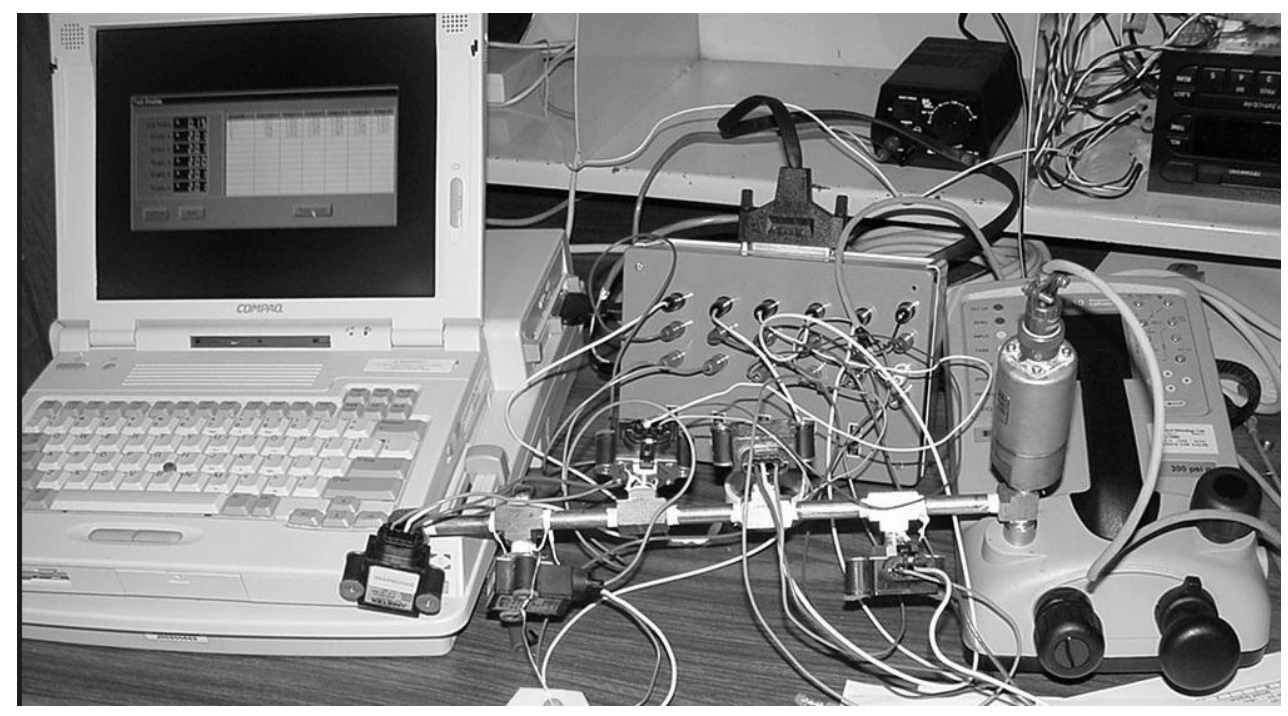

Figure 1: A pressure transducer calibration test stand

In the development, a Druck DPI-610 pressure transducer calibrator was used as the main component for testing. This device includes a high-pressure pump, a link to the personal computer, and a software package (Link-Pak) that controls the test process and provides a hard copy of the transducer calibration (QS9000 requirement) ${ }^{4}$. The test was done by first calibrating a reference transducer to the internal transducer of the Druck system to create a reference curve of the pressure voltages. The system was then set up to accommodate the testing of five transducers at once. The pressure voltage curve measured from each transducer was then compared to the reference curve which had to be within a certain tolerance range to pass as reusable. 
This project involved circuit design for analog signal amplification and A/D conversion, system integration for data acquisition and testing, and computer programming for curve fitting and data analysis. The undergraduate research assistant initiated her participation in the project by learning the system and getting familiar with the testing equipment. During the project development, the student was responsible for circuit design, programming and running the tests. One feature that indicates the complexity of this project is the calibration/functionality check of a mass airflow sensor. In order for the system to provide a known airflow through the sensor, pulse counting of the sensor output would be required. For this part of the test, the student was also responsible for communicating with the engineers in the Metrology Lab at General Motor's Milford Proving Grounds to obtain necessary information and technical support. These discussions with the engineers provided opportunities for the student to gain industrial experience required for future employment.

The project was funded in the Fall 2001 semester, with a duration of two semesters. The student graduated in May 2002, and is currently working for the GM Milford Proving Grounds in Milford, Michigan.

b. Computer-based data acquisition for audio equipment standardization

The objective of this project was to program and implement an Audio Equipment Standardized test station in LabVIEW. Such a station would provide specifications of the audio equipment, such as the Total Harmonic Distortion (THD) and Signal-to-Noise ration (S/N) recommended by IEEE and ASE.

This project involved intensive LabVIEW programming. The student initiated his participation in the project by getting a solid understanding of the LabVIEW G programming language. To simplify the development, the example VI called SINAD, provided in LabVIEW $6 \mathrm{i}$, was used as a platform to initiate the implementation of the test station. SINAD stands for signal-to-noise and distortion ratio. Figure 2 shows the front panel of the SINAD VI.

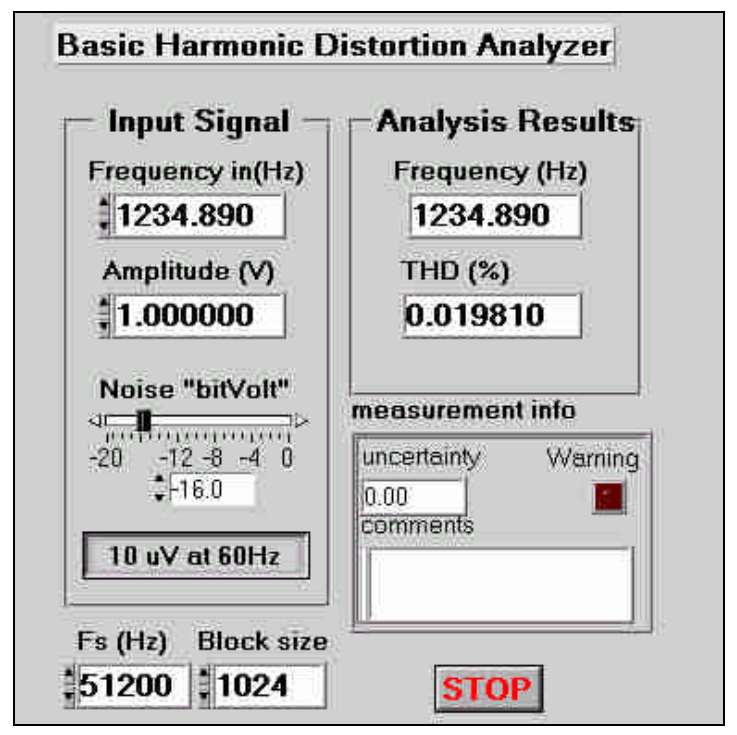

Figure 2: Front panel of the SINAD VI

"Proceedings of the 2003 American Society for Engineering Education Annual Conference \& Exposition Copyright (C) 2003, American Society for Engineering Education" 
The THD is a form of non-linearity that causes high frequency harmonics to be added to the input signal. To test for this, the input signal had to be a high purity sinusoidal wave. Typical audio signal has a frequency range from $100 \mathrm{~Hz}$ to $4 \mathrm{KHz}$, but the harmonics can extend to much higher frequencies. In THD measurement, the primary interest is to measure the harmonic frequencies in relation to the base frequency. In order to obtain an accurate THD measurement, a data acquisition board (PCI-MIO-16XE DAQ) of 100K samples per second, for 16 bit data samples was used for data acquisition. This allowed THD up to $50 \mathrm{KHz}$ to be measured. In order to use a sampling rate at this speed with a continuous waveform, a circular computer memory buffer was included in the data acquisition system, and the issue of the VI's ability to retrieve data from the buffer at this higher rate was addressed.

This project started in January 2002, and was completed in three semesters. The student who participated in this project is expecting to graduate in May 2003 with a BS degree major in Electrical Engineering Technology and Computer Science minor. He is currently working as an engineering co-op in Automotive Electrical Design Systems in Detroit, Michigan.

\section{c. Implementation of lock-in amplifier using LabVIEW virtual instrument}

The objective of this project was to implement the concept of the lock-in amplifier using LabVIEW virtual instrument and investigate its properties of noise reduction and phase diction in digital signal processing. The lock-in amplifier, sometimes referred to as the phase-sensitive signal detector, is well known for its ability in detecting and measuring weak signals from noisy environments. It has been show that the lock-in amplifier can detect a weak signal immersed down as low as $60 \mathrm{~dB}$ below the noise. Figure 3 shows the block diagram of a typical lock-in amplifier circuit.

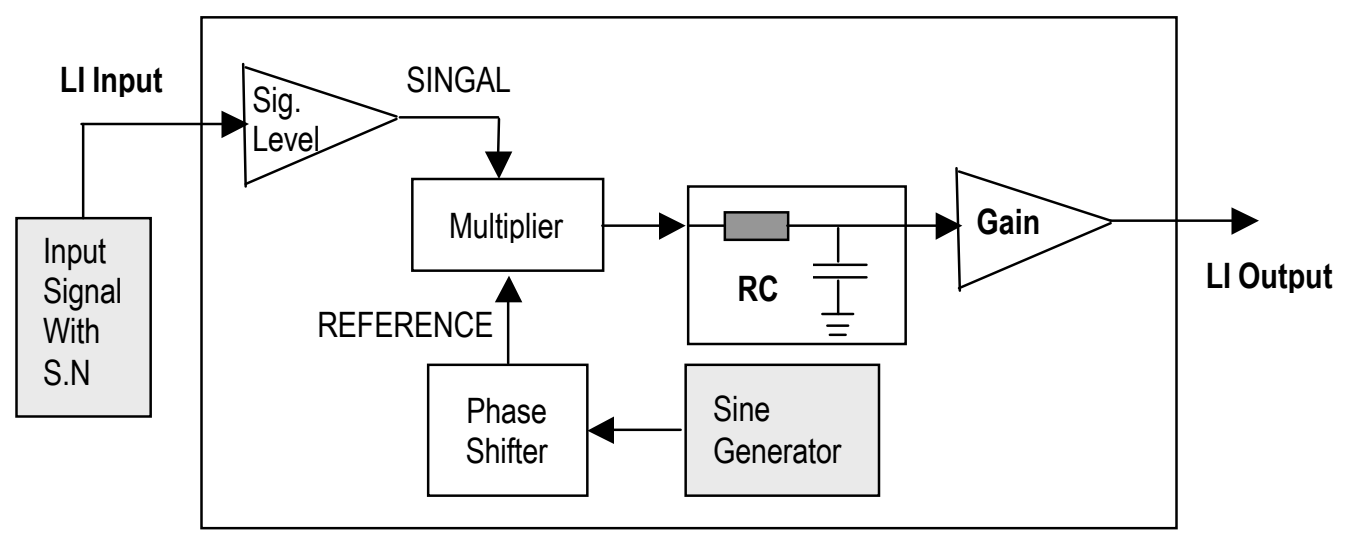

Figure 3: Block diagram of a typical lock-in amplifier

The student was responsible for programming LabVIEW VI to perform the operations of the lock-in amplifier and examining the frequency response, processing speed and phase sensitivity of the digital amplifier. The LabVIEW program consists of three major components: (1) Data Acquisition - for obtaining continuously updated and buffered analog input at the maximum sample-rate of 100k samples/sec, (2) Signal Processing - for performing crosscorrelation functions to implement phase-sensitive signal detection, and (3) User Interface - for 
adjusting amplifier parameters and the reference frequency. The feasibility of implementing the more modern lock-in analyzer in the LabVIEW environment was also investigated. The main functional difference between the lock-in analyzer and the lock-in amplifier is that the analyzer does not require the phase of the reference signal to be set manually. This makes usage of the Analyzer much simpler than that of the Amplifier.

The concept of the lock-in amplifier for phase-signal detection is based on the crosscorrelation of two sinusoidal signals. Given the local reference signal, $f_{R}(t)=a \cdot \sin \left(\Phi_{R} t\right)$, and the input signal, $f_{S}(t)=b \cdot \sin \left(\bar{\varpi}_{S} t+\phi\right)$, the cross-correlation is given by

$$
R=\frac{1}{n T} \int_{0}^{n T} f_{s}(t) \cdot f_{R}(t) \cdot d t
$$

where $T=2 \pi / \sigma_{R}$ is the period of the local reference signal. The main research problem for this project was therefore concentrated in the study and implementation of this equation.

Theoretically, the integration time, $n T$, must be set as long as possible. When implementing this in LabVIEW, the buffer size of the data acquisition system must be considered. Another point of research was to calculate or measure the time increment, $d t$, for integration. This value must be determined based on data sampling rates and processing speed, as well as the accuracy the integration output required.

This project was funded in the Summer 2002 semester, with a duration of three semesters. The student who participated in this project is expecting to graduate in May 2003. He has decided to continue graduate study for a MSET degree.

\section{Impact of undergraduate research experiences}

The engineering technology program, because of its applied nature, provides students with a large variety of research topics. Our experiences show that, with a little guidance from the faculty, undergraduate students can be good researches. To the students, the impact of undergraduate research experiences is measurable in several dimensions. The research experiences provide them exposure to the modern technology and equipment. The one-to-one discussions and communication between professors and the students has increased confidence levels of the students. They also gain valuable technical knowledge by conducting literature search, industry visits, and discussions with engineers and other technical professionals. Through the experiences of independent research, students are better prepared in the areas of critical thinking and life-long learning. This encourages the students to pursue graduate studies. At the same time, the contribution to this research project has strengthened their resume. Consequently, it greatly increases their potential for employment. The three students mentioned previously, one was recruited by local industry immediately after graduation, one obtained a coop opportunity and one decided to pursue graduate degree, are good examples of these results. We consider this as a mean for the assessment of the undergraduate research program. In the future, we will consider increasing the number of student participants on the project. This may 
add the depth to the experiment as students could work in teams on collaborative and cooperative learning.

\section{Conclusions}

This paper presents three undergraduate research projects for engineering technology students. These projects have remained as successful examples at the university for the College of Engineering and to the Division of Engineering Technology students in particular. They also reflect very well on the hands-on experience of engineering technology students. The WSU Undergraduate Research Program continues to be university wide active program. Securing NSF and other funding for the REU site establishment are under progress. Attributed to the success component of these projects, the key elements will be offered for incorporation in the other REU sites at WSU.

\section{Acknowledgements}

The authors would like to thank the WSU Undergraduate Research Council for the supports of undergraduate research program.

\section{Bibliography:}

1. W. D. Jemison, W. A. Hornfeck and J. P. Schaffer, "The Role of Undergraduate Research in Engineering Education," Proceedings of the 2001 ASEE Annual Conference, Session 3630, Albuquerque, NM.

2. S. Chickamenahalli, C. Barker, and A. Khodor, "Effective Research Experience of Electrical Engineering Technology Students" Proceedings of the 1998 Frontiers in Education Conference, Tempe, Vol. III, pp.11231125 .

3. N. Tsoulfanidis, "The Benefits of the Undergraduate Research Experience," Proceedings of the 1997 ASEE Annual Conference, Session 2377.

4. Druck Inc., "DPI-610 Portable Pressure Calibrator," Connecticut: Druck, 2000.

\section{CHIH-PING YEH}

Chih-Ping P Yeh received the B.S. degree in Electronic Engineering from Taiwan, the M.S. degree in Biomedical Engineering from Northwestern University at Evanston, IL, the M.S. and Ph.D. degrees in Electrical Engineering from Texas A\&M University at College Station, TX. Dr. Yeh is an Associate Professor in the Division of Engineering Technology at Wayne State University, Detroit, Michigan.

\section{JEANNIE M. DREW}

Jeannie M. Drew received her B.S. degree in Engineering Technology from Wayne State University in May 2002 and a two-year technical degree in Electronic Engineering from attended Detroit Engineering Institute in 1998. She has joined the United States Army for three years and worked in the electronics field from 1986 until 2002. Currently she works as a Project Engineer for General Motors Inc. in Milford, Michigan.

\section{CHRIS ROCKWEEL}

Chris Rockwell received his A.S. degree from Henry Ford Community College in 1999 Cum Laude. He is expecting to receive his B.S. degree in Engineering Technology with a Computer Science minor from Wayne State University in May 2003. He has been working as an Engineering Coop in Automotive Electrical Design Systems. 


\section{HAI-CHUN CHIEN}

Hai-Chun Chien is a senior student majoring in Electrical Engineering Technology in Wayne State University. He received an associate degree in Electrical Engineering from Lee-Min Technology College in Taipei, Taiwan in 1998. He has worked as a PLC programmer and a facility-protecting engineer in Gate Tech Industrial Inc. in Taiwan. His current research interest is the development and design of PC-Based discrete PID controller using LabVIEW. 